\title{
Do Gaming Experience and Prior Knowledge Matter When Learning with a Gamified ITS?
}

\author{
Faiza Tahir \\ Department of Computer science and \\ Software engineering \\ University of Canterbury, \\ New Zealand \\ faiza.tahir@pg.canterbury.ac.nz
}

\author{
Antonija Mitrovic \\ Department of Computer science and \\ Software engineering \\ University of Canterbury \\ New Zealand \\ tanja.mitrovic@canterbury.ac.nz
}

\author{
Valerie Sotardi \\ School of Edicational \\ Studies and Leadership \\ University of Canterbury \\ New Zealand \\ valerie.sotardi@canterbury.ac.nz
}

\begin{abstract}
Gamification has gained much popularity, due to its positive effects on learner engagement and motivation in online learning environments. However, there is still insufficient understanding of factors, including personal traits, which affect learning, as well as studies focusing on learning behaviors which can be targeted by gamification. This paper investigates the causal effects of gamification on student learning outcomes, and the role of the students' background knowledge and prior gamification experience in the relationship. The context of our study is SQLTutor, an intelligent tutoring system. Although we found no evidence of improvement in learning outcomes of the gamified group, the low prior knowledge students who received badges had higher time-on-task, made more attempts on problems and received more hints during interaction with the system. We also found that students who had previous gamification experience spent more time on problem solving as compared to those who had no prior gamification experience.
\end{abstract}

Keywords-gamification, badges, intelligent tutoring system, goals, previous gaming experience, learning outcomes

\section{INTRODUCTION AND BACKGROUND}

Gamification is defined as "the use of game design elements in non-gaming environments" [1]. It combines the enjoyment of games with the utility of a system to elevate user motivation [2]. The theory of gamified learning, proposed by Landers [3], identifies two theoretical paths through which gamification affects learning. In the first path, game elements influence learning behaviors which influence learning outcomes. In the second path, the influenced learning behavior moderates the relationship between learning content and learning outcomes.

There is very little research on gamification in Intelligent Tutoring Systems (ITSs). Abramovich and colleagues [4] awarded badges for participation and skill mastery, and reported that gamification increased topic interest but negatively influenced learning. Long and Aleven [5] awarded badges and stars while solving problems in Lynette, and reported partial positive effects on learning outcomes. However, both systems tutor middle school students, who usually are eager for awards and badges and none of them explored the influence of students' prior experience in gamification. This study presents the first empirical evidence of the gamification effects on university students in the context of SQL-Tutor, a mature ITS for teaching problem solving in Structured Query Language (SQL) [6]. Our motivation for this study is to explore the benefits of gamification on learning outcomes directly or by influencing some learning behaviours and the influence of prior knowledge and gamification experience on learning outcomes.

\section{Study DESIGN AND PROCEDURE}

SQL-Tutor has been used in database courses at the University of Canterbury since 1998, as well as by students worldwide. The system supports problem solving in SQL by providing over 300 problems defined on thirteen databases. Students can select problems by themselves or request the most appropriate problem from the system, which is selected adaptively. While solving a problem, the student has to fill in the clauses of the select statement as required by the problem statement. The system provides six types of feedback upon submission, ranging from simple feedback to complete solution. The system tracks the student's actions and maintains the model of the student's knowledge.

We used the Landers theory of gamified learning as the framework for our study. We selected goals, assessment, and challenges as game elements, and implement them in the form of 13 badges, with each having a specific wining condition. Goals are implements as the wining condition of badges. Assessment is implemented as an optional quiz, and challenges are introduced by providing three complex problems per day as a daily challenge. Students received badges on either accomplishing a goal, solving daily challenges or by attempting the quiz. We divided the badges into three different levels: primary, classic, and elite (Table 1). Primary badges are given to students to capture their attention when they first interact with the system. For example, the High flyer badge is given for solving three consecutive problems. Classic badges emphasize practicing with the system regularly; for example, the Scholar badge is awarded for solving 5 problems for five consecutive days etc. The last level consists of Elite badges, which have the purpose of engaging learners with the system for a long period. An example badge fro the elite category is Human dynamo, for solving at least five problems for ten days in a row. Students can view the achieved and unaccomplished badges on the badge page, which can be accessed via "view badges" button available on the problem-solving interface, the student model page, the introduction page of the system, and on the badge wining notification. 
TABLE I. DEFINITION OF BADGES

\begin{tabular}{|c|c|c|}
\hline Group & Badge & Condition \\
\hline \multirow{5}{*}{ Primary } & Go getter & Completing the first problem \\
\hline & High flyer & 3 problems in one session \\
\hline & Achiever & 5 problems in a day \\
\hline & Activist & $\begin{array}{l}5 \text { problems without complete } \\
\text { solution }\end{array}$ \\
\hline & Leader & $\begin{array}{l}\text { problem with the "Group by" } \\
\text { clause }\end{array}$ \\
\hline \multirow{4}{*}{ Classic } & Energy house & 6 problems in a row \\
\hline & Scholar & $\begin{array}{l}5 \text { problems/day for } 5 \text { consecutive } \\
\text { days }\end{array}$ \\
\hline & Fireball & 10 problems in one day \\
\hline & Champion & First daily challenge \\
\hline \multirow{4}{*}{ Elite } & Genius & Attempting the quiz \\
\hline & Human dynamo & 5 problems/day for 10 days \\
\hline & Einstein & 5 daily challenges over 2 weeks \\
\hline & Live-Wire & 5 problems per day for 20 days \\
\hline
\end{tabular}

Out of 198 students enrolled in the course, 77 consented to participate in the study ( $25 \%$ female, $62 \%$ male, $13 \%$ others). At the start of the study, the students completed a short questionnaire, asking about their previous experience on gamification. They were then randomly allocated to the experimental condition where they interacted with the gamified version of the ITS (experimental $=42$ ), or the non-gamified version of system (control $=35$ ). At the start of the first session, students received a pre-test to estimate their prior knowledge. The pre-test consists of nine questions (1 mark for each question), which were the combination of true-false (2) and multi-choice questions (7). Students could use the ITS whenever they wanted over the period of four weeks. At the end of the study, they received a post-test (similar to the pre-test) to assess their learning.

We made the following research hypotheses based on the results from literature and our own experience. We expect that the experimental group will be motivated by badges and learn more than the control group (H1). We expected that low prior knowledge students would engage more with the system in the gamified condition (H2). We were also interested to investigate the effects of previous gamification experience, and expected that previous gamification experience would moderate the effects of badges in the study (H3).

\section{RESULTS}

The average scores on the pre/post-test were $58.73 \%$ (sd = $26.05)$ and $69.05 \%(\mathrm{sd}=25.9)$ respectively. Only 28 students completed the post-test, as it was not mandatory and was administered two days before the major course test. There is another measure in the system called slevel, which is the current level of the student based on the number of completed problems. Slevel ranges from 1 to 9 . The average slevel for all students were $3.56(\mathrm{sd}=1.66)$ at the end of their interaction with the ITS. The average number of days (Active days) students interacted with the system was $3.39(\mathrm{sd}=2.69)$ during four weeks, and the average time spent in the system was 260 minutes ( $\mathrm{sd}=$ 243mins). During this time, students solved on average 37.47 problems $(\mathrm{sd}=34.74) .66 \%$ of experimental and $57 \%$ of the control group students reported prior experience of gamification.
Table 2 presents the statistics for the two groups. There is no significant difference on the pre-test scores, showing both groups have similar levels of pre-existing knowledge. There were also no significant differences on the time and the number of attempted/solved problems. For calculating the normalised learning gain, we consider only those students who completed both tests $(\operatorname{Exp}=17$, Control $=11)$. There was no significant improvement between pre/post-test scores for the control (mean $=.023, \mathrm{sd}=1.15)$ and experimental group $($ mean $=-0.068, \mathrm{sd}=$ 2.29) group. Therefore, H1 was not supported.

TABLE II. SUMMARY STATISTICS: MEAN (SD)

\begin{tabular}{|l|c|c|}
\hline & Experimental (42) & Control (35) \\
\hline Pre-test \% & $59.52(24.02)$ & $57.78(28.62)$ \\
\hline Time-on-task (min) & $288.40(302.02)$ & $225.94(143.44)$ \\
\hline Active Days & $3.33(3.09)$ & $3.46(2.13)$ \\
\hline Attempted problems & $42.26(42.75)$ & $37.34(26.94)$ \\
\hline Solved Problems & $39.33(40.99)$ & $35.23(25.72)$ \\
\hline Student level & $3.31(1.62)$ & $3.86(1.68)$ \\
\hline Post-test \% & $\mathrm{n}=17,67.97(26.32)$ & $\mathrm{n}=11,70.71(26.42)$ \\
\hline
\end{tabular}

To evaluate $\mathrm{H} 2$, we divided students based on their pre-test scores. Those who scored more than the median (5) were labelled as High Prior Knowledge (HPK) and others as Low Prior Knowledge (LPK). We conducted Mann-Whitney U test to compare the groups. No differences were found on HPK students from the control and experimental groups.

TABLE III. STATISTICS FOR LOW PRIOR KNOWLEDGE GROUP: MEAN (SD)

\begin{tabular}{|l|c|c|}
\hline \multicolumn{1}{|c|}{ Low Prior Knowledge } & Control (13) & Experimental (13) \\
\hline Pre-test \% & $26.4(16.11)$ & $30.7(14.44)$ \\
\hline *Time-on-task (min) & $178.31(98.25)$ & $349.77(230.48)$ \\
\hline Active days & $2.92(1.66)$ & $4.15(3.39)$ \\
\hline Problems Solved & $23.38(16.62)$ & $40.92(33.58)$ \\
\hline$*$ Attempts & $103.77(61.92)$ & $203.85(138.50)$ \\
\hline$*$ Hints & $188.38(109.16)$ & $391(261.05)$ \\
\hline *p $<.05$ & &
\end{tabular}

Table 3 presents the results of LPK students in both groups. We found no significant differences on the pre/post-test scores. The LPK students from the experimental group spent significantly more time with system $(\mathrm{U}=134, \mathrm{p}<.05)$, saw more hints $(\mathrm{U}=127, \mathrm{p}<.05)$, and had more attempts on problems $(\mathrm{U}=124.5, \mathrm{p}<.05)$ than LPK students from the control group. These results indicate that gamification may influenced behaviours of LPK students, and motivated them to interact more with the system. This confirms our Hypothesis 2.

To investigate the relationship of students' previous experience in gamification (GE) with the badges and its subsequent effects on time-on-task and slevel, we developed the model shown in Fig. 1. We take badges as an independent variable, time-on-task as a mediator and slevel as the dependent variable in the model. We added GE (Yes $=1$, No $=0)$ as a dichotomous moderating variable. To evaluate this model, first we regressed slevel on both time-on-task and badges. Results show that time-on-task is a significant predictor of slevel $(\beta=$ $.50, \mathrm{p}=.01$ ), but the number of badges is not a significant 
predictor $(\mathrm{p}=.06)$. However, the number of badges is a significant predictor of time-on-task $(\beta=.64, \mathrm{p}<.001)$.

To examine the effects of GE in the established mediating model, we investigate the moderating effects of GE on badges and time-on-task. The result shows the interaction term between badges and GE significantly and positively influences the time on task $(\mathrm{t}=2.33, \mathrm{p}=.02)$. That relationship is significant only when $\mathrm{GE}=1(\mathrm{t}=5.59, \mathrm{p}<.001)$, but not when $\mathrm{GE}=0$. This indicate that badges may helped only those students who had prior gamification experience. As evident from Fig 2, those who had no prior gamification experience spent maximum 200 minutes (mean time-on-task) with the system. On the other hand, those with previous gamification experience spent on average 370 minutes with the system.

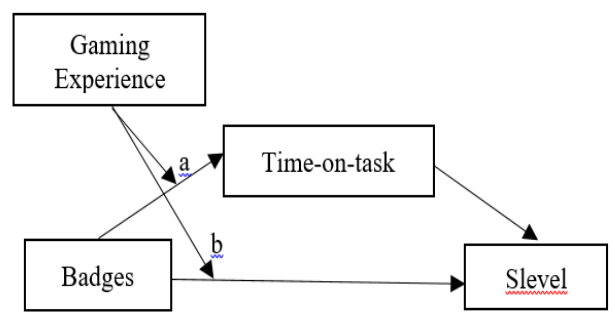

Figure 1. The moderated-mediation model, with gamification experience as a moderator

The total effect model shows the indirect effect of time-ontask is significant between badges and slevel [.0272, .4108]. This means that although GE increases time-on-task when combined with badges, in the absence of GE badges still have

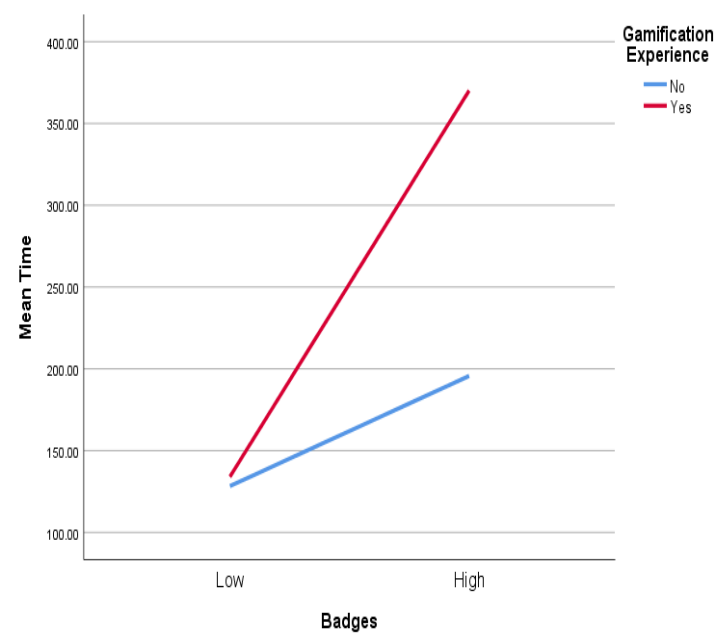

Figure 2. Relationship between badges and time-on-task when student had GE or no GE effect on student time-on-task, which influences their slevel. The index of moderated mediation confirmed the moderatedmediation effect [.0864, .7859]. This supports our hypothesis 3 .

\section{CONCLUSIONS}

In this study, we investigated the effects of gamification on learning and influence of demographic factors on gamification in context of an intelligent tutoring system. We introduced three types of badges in the system, each with different achieving criteria. The results show no difference between the experimental and control groups on student learning outcomes. However, our results indicate that gamification increased the motivation of the low prior knowledge students in the experimental group, who interacted with SQL-Tutor for a significantly higher time, made significantly more attempts on problems, and received significantly more hints than their counterparts from the control group. We also found that students who had previous experience with gamification interacted with the ITS for significantly longer time when they received badges, as compared to those who had no previous experience of gamification. Furthermore, time-on-task significantly mediates the relationship between badges and slevel.

There are two major limitations of this study. Our population size was small. The possible reason could be because the use of system was completely voluntary. The second limitation is that only $54 \%$ of the participants in the experimental condition accessed the badge page. The possible explanation for this could be the design of badges, which failed to catch the attention of learners.

\section{REFERENCES}

[1] Deterding, S., The lens of intrinsic skill atoms, "A method for gameful design," Human-Computer Interaction, 2015. 30(3-4), p. 294-335.

[2] Koivisto, J. and J. Hamari, "The rise of motivational information systems: A review of gamification research," International Journal of Information Management, 2019, 45, p. 191-210.

[3] Landers, R.N., "Developing a theory of gamified learning: Linking serious games and gamification of learning," Simulation \& gaming, 2014, 45(6), p. 752-768.

[4] Abramovich, S., Schunn, C., \& Higashi, R. M, "Are badges useful in education? It depends upon the type of badge and expertise of learner," Educational Technology Research and Development, 2013, 61(2), 217232.

[5] Long, Y., \& Aleven, V, “Gamification of joint student/system control over problem selection in a linear equation tutor," In International conference on intelligent tutoring systems, 2014, pp. 378-387, Springer, Cham

[6] Mitrovic, A, "An intelligent SQL tutor on the web," International Journal of Artificial Intelligence in Education, 2003, 13(2-4), 173-197. 